\title{
Terahertz radiation driven chiral edge currents in graphene
}

\author{
J. Karch,${ }^{1}$ C. Drexler, ${ }^{1}$ P. Olbrich, ${ }^{1}$ M. Fehrenbacher, ${ }^{1}$ M. Hirmer,${ }^{1}$ M. M. Glazov ${ }^{2}$ \\ S. A. Tarasenko, ${ }^{2}$ E. L. Ivchenko, ${ }^{2}$ B. Birkner, ${ }^{1}$ J. Eroms, ${ }^{1}$ D. Weiss,${ }^{1}$ R. Yakimova,${ }^{3}$ \\ S. Lara-Avila, ${ }^{4}$ S. Kubatkin, ${ }^{4}$ M. Ostler,${ }^{5}$ T. Seyller, ${ }^{5}$ and S. D. Ganichev ${ }^{1}$ \\ 1 Terahertz Center, University of Regensburg, 93040 Regensburg, Germany \\ 2 Ioffe Physical-Technical Institute, Russian Academy of Sciences, 194021 St. Petersburg, Russia \\ 3 Linköping University, S-58183 Linköping, Sweden \\ 4 Chalmers University of Technology, S-41296 Göteborg, Sweden and \\ 5 University of Erlangen-Nürnberg, 91058 Erlangen, Germany
}

(Dated: August 18, 2018)

\begin{abstract}
We observe photocurrents induced in single layer graphene samples by illumination of the graphene edges with circularly polarized terahertz radiation at normal incidence. The photocurrent flows along the sample edges and forms a vortex. Its winding direction reverses by switching the light helicity from left- to right-handed. We demonstrate that the photocurrent stems from the sample edges, which reduce the spatial symmetry and result in an asymmetric scattering of carriers driven by the radiation electric field. The developed theory is in a good agreement with the experiment. We show that the edge photocurrents can be applied for determination of the conductivity type and the momentum scattering time of the charge carriers in the graphene edge vicinity.
\end{abstract}

PACS numbers: 73.50.Pz, 72.80.Vp, 81.05.ue, 78.67.Wj

The "bulk" transport properties of graphene have been studied intensively in recent years and yielded insight into the half-integer and fractional quantum Hall effect, phase-coherent effects or spin transport on the micrometer scale, to name a few examples [1, 2]. While the details of each of those effects depend crucially on the linear dispersion relation of graphene and its specific material properties, most of the transport phenomena have already been studied in other two-dimensional systems. Graphene edges, on the other hand, were predicted to show insulating or metallic, even magnetic behavior, depending on the crystallographic orientation and edge chemistry. In scanning tunneling experiments, an enhanced edge density of states was shown [3, 4] and Raman scattering experiments provided evidence for the dependence of scattering mechanisms on the edge orientation [5, [6]. In transport experiments edge effects are usually masked by bulk properties, nonetheless the graphene edges are expected to play a crucial role in the electronic properties of graphene-based nanoscale devices.

Here, we present an opto-electronic method to uniquely distinguish edge from bulk scattering by exploring edge photocurrents in graphene samples illuminated by terahertz $(\mathrm{THz})$ radiation. For circularly polarized light the edge current is observed to form a vortex winding around the edges of the square-shaped samples. Its direction reverses upon switching the radiation helicity from left- to right-handed. Evidently, the photocurrent is caused by the local symmetry breaking at the sample edges resulting in an asymmetric scattering of carriers driven by the radiation electric field. It gives rise to a directed electric current along the sample boundary in a narrow stripe of width comparable to the mean free path. We show that the photocurrent measurements provide direct access to electron scattering at the graphene edges and allow to map the variation of scattering times along the edges.

We investigated two types of single-layer graphene samples: (i) large-area epitaxial graphene prepared by high-temperature $\mathrm{Si}$ sublimation of $4 \mathrm{H}$ and $6 \mathrm{H}$ polytypes of semi-insulating $\mathrm{SiC}$ substrates 7 - 9] and (ii) small area exfoliated graphene flakes [1] deposited on oxidized silicon wafers. Below, we report results on epitaxial graphene samples (labeled \#1-4H, \#2-4H, and \#3-6H) and three samples prepared from exfoliated graphene. Hall measurements indicate that the epitaxial samples are $n$-doped (due to charge transfer from $\mathrm{SiC}$ 7]) while the exfoliated samples are $p$-doped. The measured carrier density lies in the range $(2 \div 7) \times 10^{12} \mathrm{~cm}^{-2}$, the Fermi

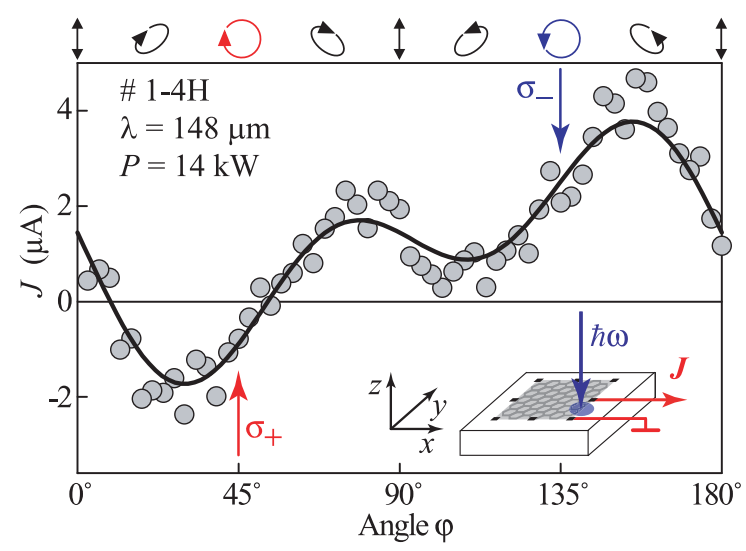

FIG. 1: Photocurrent in sample \#1-4H as a function of the angle $\varphi$ defining the light polarization. Solid line is a fit to Eq. (11) [see also Eq. (7) and discussion]. The inset shows the experimental geometry. The ellipses on top illustrate the polarization states for various $\varphi$. 


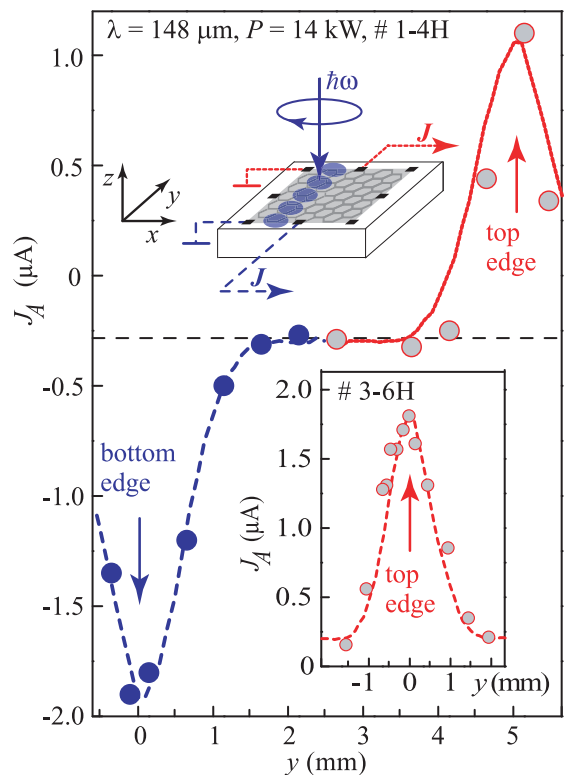

FIG. 2: Photocurrent $J_{A}$ in sample \#1-4H as a function of the laser spot position. The laser spot is scanned along $y$ and the current is picked up from two contact pairs at the top (red circles) or bottom (blue full circles) sample edges aligned along $x$ (see inset). Dashed lines represent the laser beam spatial distribution, which is measured by a pyroelectric camera, scaled to the current maximum. The bottom inset shows the scan for sample \#3-6H.

energy $E_{F}$ ranges from 200 to $300 \mathrm{meV}$ and the mobility is about $1000 \mathrm{~cm}^{2} / \mathrm{Vs}$ at room temperature. Ohmic contacts were made at samples' edges (see, e.g., inset of Fig. 11). Details on the material growth and characterization can be found in [10].

The experiments on edge photocurrents are performed applying alternating electric $\mathrm{THz}$ fields of a high power pulsed $\mathrm{NH}_{3}$ laser 11 13. operating at wavelengths $\lambda=$ $90.5 \mu \mathrm{m}, 148 \mu \mathrm{m}$ or $280 \mu \mathrm{m}$ (frequencies $f=3.3 \mathrm{THz}$, $2 \mathrm{THz}$ and $1.1 \mathrm{THz}$, respectively). The radiation induces indirect (Drude-like) optical transitions, because the photon energies are much smaller than the carrier Fermi energy. The $\mathrm{NH}_{3}$ laser generates single pulses with a duration of about $100 \mathrm{~ns}$, peak power of $P \approx 10 \mathrm{~kW}$, and a repetition rate of $1 \mathrm{~Hz}$. A typical spot diameter from 1 to $3 \mathrm{~mm}$. The beam has an almost Gaussian form, which is measured by a pyroelectric camera [14].

All experiments are performed at normal incidence of light and at room temperature. Elliptically and, in particular, circularly polarized radiation is obtained applying $\lambda / 4$ quartz plates. The resulting polarization state described by the Stokes parameters [15] $S_{1}, S_{2}$, and $S_{3} \equiv P_{\text {circ }}$ is directly related to the angle $\varphi$ between the initial linear polarization of the laser light along the $y$ axis and the plate optical axis. The experimental geometry is shown in Figs. 1, 2. and 3. The current is measured via the voltage drop across a $50 \Omega$ load resistor.
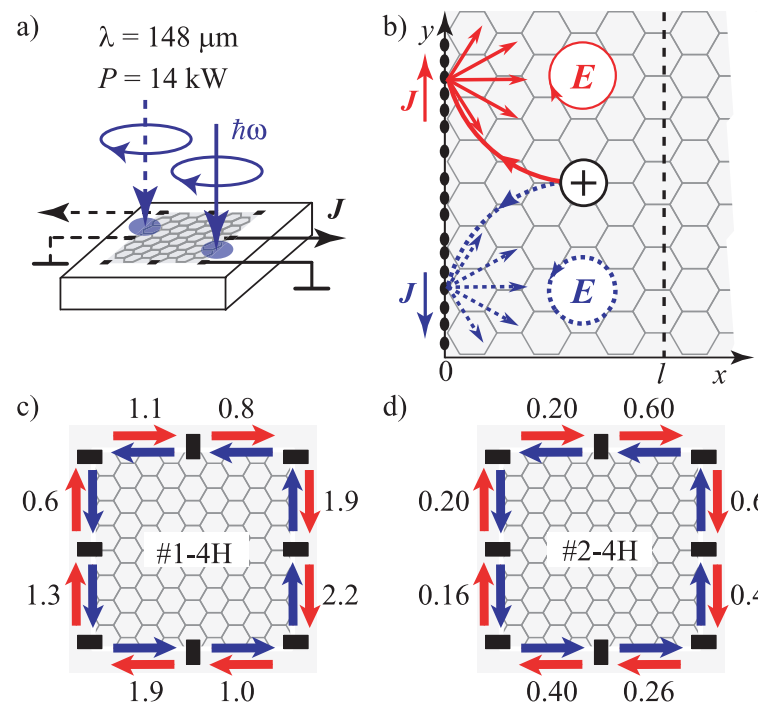

d)

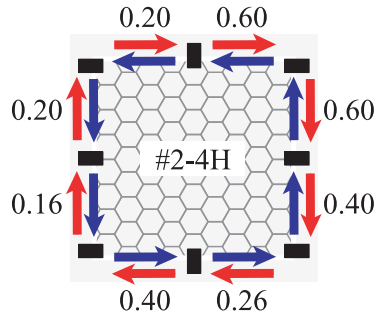

FIG. 3: (a) Experimental geometry for the study of edge photocurrents. (b) Schematic illustration of the edge current generation. The electric field of circularly polarized radiation rotates clockwise or counterclockwise resulting in a circular motion of carriers, which is sketched by red and blue trajectories, respectively. Our theoretical model, see Eq. (7), shows that the circular edge current stems from carriers moving towards the edge. It is due to the second order $\boldsymbol{E}$-field correction to the distribution function and involves the retardation of the electron motion with respect to the instantaneous electric field. Switching the radiation helicity reverses the motion direction and, consequently, the electric current. (c) and (d) photocurrent topology. Red and blue arrows show the current direction for $\sigma_{+}$and $\sigma_{-}$polarizations, respectively. Numbers indicate the photocurrent amplitude $J_{A}$ in microampers.

Illumination of the edge of unbiased large-area samples between any pair of contacts results in a photocurrent. By contrast, if the laser spot is moved toward the center the signal vanishes. The detected signal depends strongly on the radiation polarization, Fig. 1. The principal observation is that for right- $\left(\sigma_{+}\right)$and left-handed $\left(\sigma_{-}\right)$polarizations, i.e., for $\varphi=45^{\circ}$ and $135^{\circ}$, the signs of the photocurrent $J$ are opposite. The overall dependence $J(\varphi)$ is more complex. It is well described by

$$
\begin{aligned}
J(\varphi) & =J_{A} \sin 2 \varphi+\left(J_{B} / 2\right) \sin 4 \varphi-J_{C} \cos ^{2} 2 \varphi+\xi \\
& =J_{A} P_{\text {circ }}(\varphi)+J_{B} S_{2}(\varphi)+J_{C} S_{1}(\varphi)+\xi
\end{aligned}
$$

and corresponds to the superposition of the Stokes parameters with different weights. The first term given by the coefficient $J_{A}$ is just proportional to the radiation helicity, whereas the second $\left(J \propto J_{B}\right)$ and third $\left(J \propto J_{C}\right)$ terms change with degree and orientation of the linear polarization. Note that the observed offset $\xi$ is usually smaller or comparable to $J_{A}, J_{B}$, and $J_{C}$ (see Fig. 1). In our present study we focus on the helicity driven photocurrent $J_{A}$. This is the only contribution which reverses the current direction upon switching the radiation helicity from $\sigma_{+}$to $\sigma_{-}$. We also note that, for circu- 


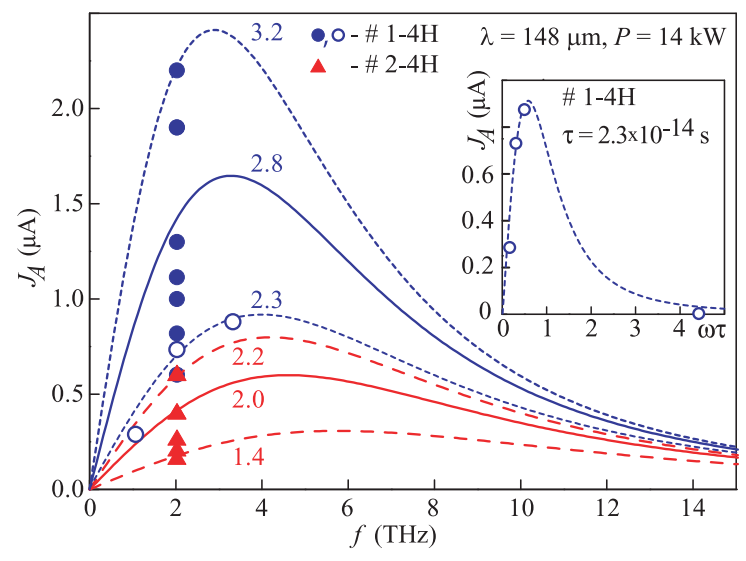

FIG. 4: Photocurrent $J_{A}$ measured at $2 \mathrm{THz}$ for different edge segments [see Figs. 36 and 3 for circles and triangles, respectively]. Lines are fits to Eq. (7). The fitting parameters $\tau / 10^{-14}$ s for sample \#1-4H and \#2-4H are indicated by numbers. The inset shows the measured circular photocurrent $J_{A}(\omega \tau)$ at one of the edge segments of sample \#1-4H (open circles) together with the fit after Eq. (7). Data point at $\omega \tau=4.4$ is obtained applying pulsed $\mathrm{CO}_{2}$ laser.

larly polarized light $\left(P_{\text {circ }}= \pm 1\right.$ and $\left.S_{1}=S_{2}=0\right)$ and $\xi=0$, the current is solely determined by the first term in Eq. (11). Therefore, it would be sufficient to measure the response to circularly polarized radiation. However, to increase the accuracy, we always measured the whole polarization dependence, like the one shown in Fig. [1. and extracted $J_{A}$ by fitting Eq. (11) to the data.

To prove that the photocurrent is caused by illuminating the graphene edges, we scanned the laser spot across the sample along the $y$-axis. The signal was picked up from a pair of contacts at the sample top and bottom edges aligned along the $x$-axis. The experimental geometry and the photocurrent $J_{A}$ versus the spot position are shown in Fig. 2. The current reaches its maximum for the laser spot centered at the edge and rapidly decays with the spot moving. Comparison of $J_{A}(y)$ with the independently recorded laser profile (dotted line) shows that the signal just follows the Gaussian intensity profile. This observation unambiguously demonstrates that the photocurrent is caused by illuminating the sample edges. Moreover, Fig. 2 reveals that the helicity driven current $J_{A}$ changes its sign for opposite edges.

The above results show that the current direction at a specific edge depends on the light helicity. To check this in more detail, we investigated currents excited by circularly polarized radiation for different pairs of contacts. Here, the laser spot is always centered between the contacts, see Fig. 3a. The current direction for $\sigma_{+}$ (red arrows) and $\sigma_{-}$(blue arrows) circularly polarized radiation and the magnitude of $J_{A}$ for various contact pairs are shown in Fig. 3b and 3 . The figures document a remarkable behavior of the circular edge photocurrent: it forms a vortex winding around the edges of the square shaped samples which reverses its direction upon switching from right- to left-handed. These dependencies are observed for all used wavelengths and samples. Helicity driven currents have also been observed for small area graphene flakes, see [10] for details.

The observation that a photocurrent occurs only if the laser spot is adjusted to an edge agrees with the symmetry analysis: In the ideal honeycomb lattice of graphene and for our experimental geometry, any photoelectric effect is forbidden [16], because the two-dimensional structure of graphene possesses a center of space inversion. Thus, the appearance of photocurrents at normal incidence of radiation is a clear manifestation of the symmetry reduction of the system, in our case, due to the edges. We also note that the typical photon energy $\hbar \omega \sim 10 \mathrm{meV}$ used in experiment is much smaller than the characteristic energy of carriers $E_{F} \sim 100 \mathrm{meV}$. Thus, the mechanism of current formation can be treated classically and should involve the action of the light's electric field on free carriers in the vicinity of a graphene edge.

A microscopic process actuating the edge photocurrent generation is illustrated in Fig. $3 \mathrm{~b}$. It involves the time dependent motion of carriers under the action of the electric field of circularly polarized radiation and scattering at the sample edge. We note that this mechanism is similar to that of the surface photogalvanic effect observed in bulk materials [17, 18]. The microscopic theory of edge currents is developed in the framework of the Boltzmann kinetic equation. In this approach, the electron (hole) distribution is described by the function $f(\boldsymbol{p}, x, t)$. It depends on the carrier momentum $\boldsymbol{p}$, coordinate $x(x \geq 0$ for a semi-infinite layer), time $t$, and obeys the equation

$$
\frac{\partial f}{\partial t}+v_{x} \frac{\partial f}{\partial x}+q \boldsymbol{E}(t) \frac{\partial f}{\partial \boldsymbol{p}}=Q\{f\},
$$

where $\boldsymbol{E}(t)=\boldsymbol{E}_{0} \mathrm{e}^{-\mathrm{i} \omega t}+\boldsymbol{E}_{0}^{*} \mathrm{e}^{+\mathrm{i} \omega t}$ is the electric field of the radiation, $\boldsymbol{v}=v \boldsymbol{p} / p$ is the electron velocity, $v \approx 10^{6} \mathrm{~m} / \mathrm{s}$ is the effective speed, $q$ is the carrier charge $(q=+|e|$ for holes and $-|e|$ for electrons), and $Q\{f\}$ is the collision integral. The distribution function can be expanded in series of powers of the electric field,

$f(\boldsymbol{p}, x, t)=f_{0}\left(\varepsilon_{\boldsymbol{p}}\right)+\left[f_{1}(\boldsymbol{p}, x) \mathrm{e}^{-\mathrm{i} \omega t}+\right.$ c.c. $]+f_{2}(\boldsymbol{p}, x)+\ldots$,

where $f_{0}\left(\varepsilon_{\boldsymbol{p}}\right)$ is the equilibrium distribution function with $\varepsilon_{\boldsymbol{p}}=v p$ being the electron energy, $f_{1} \propto|\boldsymbol{E}|$, and $f_{2} \propto|\boldsymbol{E}|^{2}$. The first order in $\boldsymbol{E}$ correction to the distribution function oscillates with frequency $\omega$ and does not contribute to a $d c$ current. The directed electric current along the structure edge is, therefore, determined by the second order $\boldsymbol{E}$-field correction $f_{2}$ and given by

$$
J_{y}=4 q \int_{0}^{\infty} d x \sum_{\boldsymbol{p}} f_{2}(\boldsymbol{p}, x) v_{y} .
$$

The factor 4 accounts for the spin and valley degeneracy. 
We solve Eq. (2) and calculate the current, Eq. (4), for the simple form of the collision integral,

$$
Q\{f(\boldsymbol{p}, x, t)\}=-\frac{f(\boldsymbol{p}, x, t)-f_{0}\left(\varepsilon_{\boldsymbol{p}}\right)}{\tau},
$$

with $\tau$ being the scattering time, and the boundary condition at $x=0$,

$$
\begin{aligned}
f\left(p_{x}>0\right. & \left., p_{y}, 0, t\right)= \\
& -\int \frac{v_{x}^{\prime}}{2 p^{\prime}} f\left(\boldsymbol{p}^{\prime}, 0, t\right) \delta\left(\varepsilon_{\boldsymbol{p}}-\varepsilon_{\boldsymbol{p}^{\prime}}\right) \Theta\left(-v_{x}^{\prime}\right) d \boldsymbol{p}^{\prime},
\end{aligned}
$$

corresponding to diffusive scattering. In the case of a degenerate gas, the edge current takes the form (see [10] for details):

$$
\begin{gathered}
J_{y}=-\frac{q^{3} \tau^{3} v^{2}}{2 \pi \hbar^{2}\left[1+(\omega \tau)^{2}\right]}\left[\frac{10}{3} \frac{\omega \tau}{1+(\omega \tau)^{2}} \mathrm{i}\left[\boldsymbol{E}_{0} \times \boldsymbol{E}_{0}^{*}\right]_{z}+\right. \\
\left.\left(1+\frac{7}{6} \frac{1-(\omega \tau)^{2}}{1+(\omega \tau)^{2}}\right) \times\left(E_{0, x} E_{0, y}^{*}+E_{0, y} E_{0, x}^{*}\right)\right] .
\end{gathered}
$$

The helicity-driven current is given by the first term because i $\left[\boldsymbol{E}_{0} \times \boldsymbol{E}_{0}^{*}\right]_{z} \equiv-P_{\text {circ }}$ for our geometry where the light propagates along $-z$. The second term yields the current caused by linearly polarized radiation and vanishes for circular polarization. In the case of elliptically polarized light, $E_{0, x} E_{0, y}^{*}+E_{0, y} E_{0, x}^{*} \propto(1 / 2) \sin 4 \varphi=S_{2}$. Both contributions are clearly detected in the experiment and correspond to the first $\left(\propto J_{A}\right)$ and second $\left(\propto J_{B}\right)$ terms in the empirical Eq. (1), see Fig. 1 .

The helicity driven photocurrent described by Eq. (7) vanishes for zero frequency, has a maximum at $\omega \tau \simeq 0.6$ and decreases rapidly at higher frequencies. Exactly this behavior is found in experiment (see inset of Fig. 4) as we explain in more detail below. The only free parameter in Eq. (7) is the scattering time $\tau$. Corresponding data are shown in Fig. 3 where the photocurrent values measured at $2 \mathrm{THz}$ for each of the contact pairs are plotted. These data points are first compared to calculated traces of $J_{A}$ employing Eq. (7). Solid lines are calculated using the bulk values for the time $\tau$ extracted from resistivity and carrier density for samples \#1-4H $\left(\tau=2.0 \times 10^{-14} \mathrm{~s}\right)$ and \#2-4H $\left(\tau=2.8 \times 10^{-14} \mathrm{~s}\right)$. The bulk scattering times used in Eq. (7) give for some of the contact pairs already perfect quantitative agreement. For other edge segments the current deviates significantly. This is a consequence of the strongly non-linear dependence of $J_{A}$ on $\tau$. Varying $\tau$ by only $\pm 15 \%$ changes the current by $\pm 50 \%$. By fitting the photocurrent $J_{A}$ we can extract the local scattering time $\tau$ for every edge segment shown in Figs. 3 . and d). The best fits are shown by dashed lines in Fig. 4 and constitute a map of scattering times along the edge. The average value of the circular edge current scales with the sample mobility. To check the frequency dependence predicted by Eq. (7) we show in the inset of Fig. 4. $J_{A}$ vs. $\omega \tau$ for one edge segment using the extracted $\tau$. The data points are perfectly described by Eq. (7) and confirm the model.

While the magnitude of the circular edge photocurrent agrees well with theory, the expected polarity of $J_{A}$ for $n$-type graphene is opposite to the one observed. This, at first glance, surprising result agrees with results from spatially resolved Raman measurements demonstrating that edges of $n$-type graphene layers exhibit $p$-type conductivity [5, 6]. This explains the sign of the photocurrent, which is generated in a narrow edge channel comparable to the mean free path $(\approx 10 \div 20 \mathrm{~nm})$ and has opposite sign for electrons and holes, see Eq. (7). Actually, the difference in the conductivity type can be also understood from the details of the sample fabrication. It is well established that epitaxial graphene on $\mathrm{SiC}(0001)$ is $n$-doped due to charge transfer from the interfacial buffer layer (see, e.g., 7, 8]), while so-called quasi-free-standing graphene, lacking such buffer layer and sitting on a hydrogen terminated $\mathrm{SiC}(0001)$ surface, is $p$-doped [19]. Therefore, it is reasonable that the edges of epitaxial graphene, exposed to the $\mathrm{SiC}$ substrate without the interfacial layer, can be $p$-doped. This assumption is corroborated by similar reports on the transition from $n$ to $p$-type of doping at the edges of graphene flakes on $\mathrm{SiO}_{2}$, which were attributed to the difference in the work functions of graphene and the substrate [20].

To summarize, our observations clearly demonstrate that illuminating monolayer graphene edges with polarized terahertz radiation at normal incidence results in a directed electric edge current. The effect is directly coupled to electron scattering at the graphene edge and vanishes in bulk graphene. Our results suggest that circular the photocurrents can be effectively used to study edge transport in graphene even at room temperature.

We thank K. S. Novoselov, V. Lechner, S. Heydrich and V. V. Bel'kov for fruitful discussions. Support from DFG (SPP 1459 and GRK 1570), EU-ConceptGraphene, Linkage Grant of IB of BMBF at DLR, RFBR, Russian Ministry of Education and Sciences, and "Dynasty" Foundation-ICFPM is acknowledged. 


\section{SUPPLEMENTAL MATERIAL}

\section{S1. Details of the Samples}

We investigated three epitaxial samples grown on SiC. Samples \#1-4H and \#2-4H were grown by the Linköping group on a Si-terminated surface of a $4 \mathrm{H}-\mathrm{SiC}(0001)$ semiinsulating substrate (Cree Inc.) [8]. The reaction kinetics on the Si-terminated surface is slower than on the $\mathrm{C}$-face because of the higher surface energy, which fosters homogeneous and well controlled graphene formation [7]. Graphene was grown at a temperature of $2000^{\circ} \mathrm{C}$ and 1 atm Ar gas pressure resulting in monolayers of graphene atomically uniform over more than $1000 \mu \mathrm{m}^{2}$, as shown by low-energy electron microscopy [21]. Eight contacts were produced by depositing $3 \mathrm{~nm}$ of $\mathrm{Ti}$ and $100 \mathrm{~nm}$ of Au. The quadratic sample size of $5 \times 5 \mathrm{~mm}^{2}$ was achieved by oxygen plasma etching of all four edges. Hall measurements indicate that the large area samples are $n$ doped due to charge transfer from SiC [7, 8, 22 25]. The measured carrier concentration is between $3 \times 10^{12} \mathrm{~cm}^{-2}$ and $7 \times 10^{12} \mathrm{~cm}^{-2}$, the Fermi energy $E_{F}$ ranges from 200 to $300 \mathrm{meV}$ and the mobility is about $1000 \mathrm{~cm}^{2} / \mathrm{Vs}$ at room temperature. In these samples, as well as in other large-area samples, the resistance at room temperature is about 2 to $5 \mathrm{k} \Omega$.

The third epitaxial graphene sample \#3-6H, was grown by the Erlangen group on $6 \mathrm{H}-\mathrm{SiC}(0001)$ wafers (II-VI Inc.). Graphene growth was performed using sublimation growth in Ar atmosphere [7, 9]. First, polishing damage was removed by etching the substrate in 1 bar hydrogen at $1550^{\circ} \mathrm{C}$ for $15 \mathrm{~min}$. Second, graphene was grown by annealing the sample in 1 bar Ar at a temperature of $1650^{\circ} \mathrm{C}$ for $15 \mathrm{~min}$. The graphene coverage was determined by x-ray photoelectron spectroscopy (XPS). The square-shaped sample size of $4 \times 4 \mathrm{~mm}^{2}$ was achieved by mechanical cutting the edges. Both, carrier density and mobility in the $n$-type sample $\# 3-6 \mathrm{H}$ are very similar to those of the Linköping samples.

For all epitaxial graphene samples, low-temperature quantum Hall measurements reveal the high quality and homogeneity.

The exfoliated graphene samples (small-area graphene samples 4, 5, and 6) has been prepared from natural graphite using the mechanical exfoliation technique [1] on an oxidized silicon wafer. The oxide thickness of $300 \mathrm{~nm}$ allowed to locate graphene flakes in an optical microscope and to assess their thickness. We checked the reliability of this method using Raman spectroscopy and low-temperature quantum Hall measurements on similar samples [26]. The single layer graphene flakes obtained by this method were typically $p$-doped by adsorbed contaminants with carrier concentrations $p \leq 2 \times 10^{12} \mathrm{~cm}^{-2}$. The Fermi energies were $E_{F} \leq 165 \mathrm{meV}$ and the mobilities at room temperature of the order of $2.5 \times 10^{3} \mathrm{~cm}^{2} / \mathrm{Vs}$.
The flakes included in this study were all single layer with the flakes size of the order of 10 to 30 micrometers. The sample morphology was characterized by atomic force microscopy measurements under ambient conditions with the microscope in intermittent contact mode with standard silicon tips [27]. After recording the position of the flakes with respect to predefined markers, we contacted them by electron beam lithography and thermal evaporation of $60 \mathrm{~nm} \mathrm{Pd}$ electrodes. The resistance of graphene measured between various contacts was about 1 to $3 \mathrm{k} \Omega$.

\section{S2. Laser beam parameters}

In addition to the pulsed $\mathrm{THz}$ laser described in the main text, we also used a continuous-wave (cw) $\mathrm{CH}_{3} \mathrm{OH}$ laser $(\lambda=118 \mu \mathrm{m})$ with a power of $P \approx 20 \mathrm{~mW}$. In the experiments applying the $\mathrm{CH}_{3} \mathrm{OH}$ laser, the cw radiation was modulated at chopper frequencies in the range from 120 to $600 \mathrm{~Hz}$. The sign of the signal is defined as a relative phase with respect to the lock-in reference signal, which was kept the same for all measurements.

Elliptically and, in particular, circularly polarized radiation has been obtained by transmitting the laser beam, which is initially linearly polarized along the $y$-direction for the pulsed laser and along the $x$-direction for the cw laser, through $\lambda / 4$ crystal quartz plates. The resulting polarization state is directly related to the angle $\varphi$ between the initial linear polarization of the laser light and the optical axis of the plate. It is described by the Stokes parameters $S_{1}, S_{2}, S_{3}$ [15]. In particular, the dependence of the circular polarization degree, given by $S_{3}$, on the angle $\varphi$ in our experimental geometry has the form

$$
P_{\text {circ }} \equiv S_{3}(\varphi)=\sin 2 \varphi \text {. }
$$

The parameters $S_{1}$ and $S_{2}$ are given by the bilinear combinations of the polarization vector components,

$$
\begin{aligned}
& S_{1}(\varphi) \equiv\left|e_{x}\right|^{2}-\left|e_{y}\right|^{2}=-\cos ^{2} 2 \varphi, \\
& S_{2}(\varphi) \equiv e_{x} e_{y}^{*}+e_{y} e_{x}^{*}=\frac{1}{2} \sin 4 \varphi .
\end{aligned}
$$

The parameters $S_{1}$ and $S_{2}$ describe the degree of linear polarization in the coordinate axes $x, y$ and in the coordinate frame rotated about an angle of $45^{\circ}$, respectively [15]. Note that radiation is incident along $-z$ axis. The resulting polarization ellipses for the $\mathrm{cw} \mathrm{THz}$ laser for some angles $\varphi$ are sketched on top of Fig. 5. Further details on the experimental technique can be found in Ref. [28].

\section{S3. Photocurrents in small-area samples}

Helicity driven photocurrents excited at normal incidence have also been observed in small-area graphene 


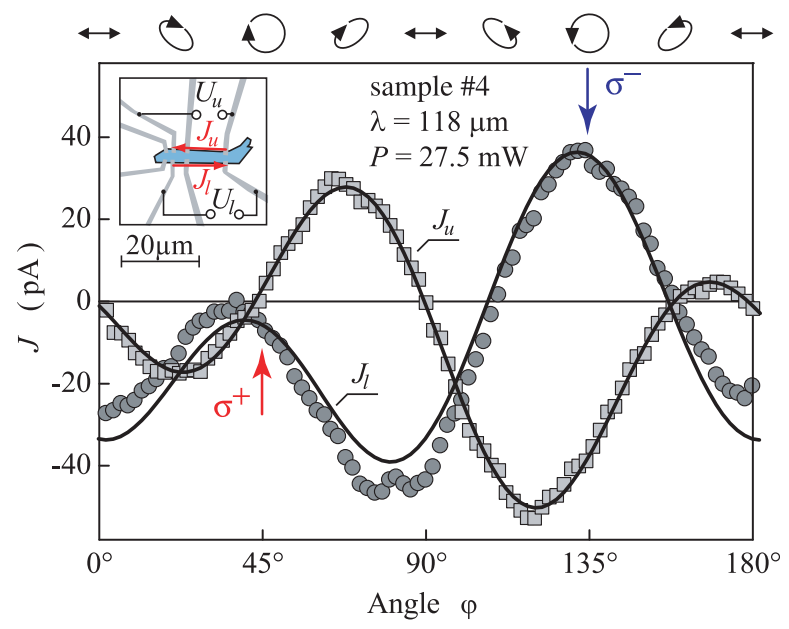

FIG. 5: Photocurrent in small area sample \#4 as a function of the angle $\varphi$. Squares and circles show the photocurrent picked up from the contact pairs at the upper $\left(J_{u}\right)$ and lower $\left(J_{l}\right)$ edges of the graphene flake, respectively (see inset). Full lines show fits to the calculated total current after Eq. (10). The inset demonstrates the experimental geometry, $U_{u}$ and $U_{l}$ are voltages recorded by lock-in amplifier. Arrows show current directions for right circularly polarized light. The signal is excited by radiation with the wavelength $\lambda=118 \mu \mathrm{m}$, power $P \approx 20 \mathrm{~mW}$ and a diameter of the laser spot about $1.5 \mathrm{~mm}$. On top, the polarization ellipses corresponding to various angles $\varphi$ are illustrated.

flakes. Examples of the current polarization dependence are shown in Fig. 5 for two different pairs of contacts. Similar to data obtained in large-area samples, it can be well fitted by Eq. (1) of the main text, which reads

$$
J(\varphi)=J_{A} P_{\text {circ }}(\varphi)+J_{B} S_{2}(\varphi)+J_{C} S_{1}(\varphi)+\xi .
$$

While photocurrents are observed in both large-area and small-area samples, the analysis of the edge photocurrents is much easier in the large-area samples. Actually only in the latter type of samples the illumination of a single edge by $\mathrm{THz}$ radiation could be realized in our experiments. Such selective excitation has enabled the accurate analyses of the edge currents. By contrast, in micron-sized exfoliated samples the spot size is much larger than the graphene flakes and the effects of different edges are superimposed.

\section{S4. Theory}

Equation (7) of the main text is obtained by expanding the distribution function $f(\boldsymbol{p}, x, t)$ in series of the electric field. To first order in the electric field, solution of Eq. (2) with the boundary condition (6) has the form

$$
\begin{aligned}
f_{1}(\boldsymbol{p}, x) & =-\frac{q \tau f_{0}^{\prime}}{1-\mathrm{i} \omega \tau}\left[\boldsymbol{E}_{0} \cdot \boldsymbol{v}-\right. \\
\left(\boldsymbol{E}_{0} \cdot \boldsymbol{v}\right. & \left.\left.+\frac{\pi}{4} E_{0, x} v\right) \exp \left(-\frac{1-\mathrm{i} \omega \tau}{v_{x} \tau} x\right) \Theta\left(v_{x}\right)\right],
\end{aligned}
$$

where $f_{0}^{\prime}=d f_{0}(\varepsilon) / d \varepsilon$. The equation for the second-order correction $f_{2}(\boldsymbol{p}, x)$ is given by

$$
v_{x} \frac{\partial f_{2}(\boldsymbol{p}, x)}{\partial x}+2 q \Re\left[\boldsymbol{E}_{0}^{*} \frac{\partial f_{1}(\boldsymbol{p}, x)}{\partial \boldsymbol{p}}\right]=-\frac{f_{2}(\boldsymbol{p}, x)}{\tau},
$$

which yields

$$
\begin{aligned}
\int_{0}^{\infty} f_{2}(\boldsymbol{p}, x) d x= & v_{x} \tau\left[f_{2}(\boldsymbol{k}, 0)-f_{2}(\boldsymbol{k}, \infty)\right] \\
& -2 q \int_{0}^{\infty} \Re\left[\boldsymbol{E}_{0}^{*} \frac{f_{1}(\boldsymbol{p}, x)}{d \boldsymbol{p}}\right] d x .
\end{aligned}
$$

By using Eq. (4) for the edge electric current and Eq. (13) we derive

$$
\begin{gathered}
J_{y}=-8 q^{3} \tau^{3} \sum_{p} \Re\left\{\frac{v_{x} v_{y} \boldsymbol{E}_{0}^{*}}{1-\mathrm{i} \omega \tau} \frac{d\left[\left(\boldsymbol{E}_{0} \cdot \boldsymbol{v}\right) f_{0}^{\prime}\right]}{d \boldsymbol{p}}\right. \\
\left.+\frac{v_{y} \boldsymbol{E}_{0}^{*}}{(1-\mathrm{i} \omega \tau)^{2}} \frac{d}{d \boldsymbol{p}}\left[\left(\boldsymbol{E}_{0} \cdot \boldsymbol{v}+\frac{\pi}{4} E_{0, x} v\right) f_{0}^{\prime} v_{x}\right]\right\} \Theta\left(v_{x}\right),
\end{gathered}
$$

where the above two contributions to the current stem from the first and second terms on the right-hand side of Eq. (13), respectively. Finally, taking into account that the electron energy and velocity in graphene are given by $\varepsilon_{\boldsymbol{p}}=v|\boldsymbol{p}|$ and $\boldsymbol{v}=v \boldsymbol{p} / p$, respectively, and assuming that the electron gas is degenerate and $\tau$ is independent of $\varepsilon_{\boldsymbol{p}}$, we obtain Eq. (7) of the main text.

The current Eq. (7) of the main text is consistent with the point-group symmetry $\mathrm{C}_{s}$ containing the mirror plane $(x, z)$. It should be noted that for elliptical polarization, in some experiments the photocurrent described by Eq. (7) is superimposed with an additional contribution proportional to $\cos ^{2} 2 \varphi$ [see the third term in Eq. (10)]. This term can be attributed to a lowering of the system symmetry to $\mathrm{C}_{1}$ showing the non-equivalence of $y$ and $-y$ directions, e.g., due to (i) inhomogeneous photoexcitation, (ii) macroscopic roughness of the investigated edges, (iii) non-equivalence of contacts, etc. The symmetry reduction hinders the edge photogalvanic currents under study and complicates their analysis. However, the obstacle can be easily overcome applying circularly polarized radiation, like used in the present work. Indeed, as addressed above the edge currents driven by circularly polarized light change their signs upon variation of the radiation helicity. By contrast, other current contributions caused by the additional symmetry lowering are insensitive to the radiation helicity and vanish for circularly polarized radiation. 
[1] K. S. Novoselov et al., Science 306, 666 (2004).

[2] S. Das Sarma et al., Rev. Mod. Phys. 83, 407 (2011).

[3] Y. Nimi, et al. Phys. Rev. B 73, 085421 (2006).

[4] K. A. Ritter and J. W. Lyding Nature Mat. 8, 235 (2009).

[5] C. Casiraghi et al., Nano Lett. 9, 1433 (2009).

[6] S. Heydrich et al., Appl. Phys. Lett. 97, 043113 (2010).

[7] K. V. Emtsev et al., Nature Mat. 8, 203 (2009).

[8] A. Tzalenchuk et al., Nature Nanotech. 5, 186 (2010).

[9] M. Ostler et al., Phys. Stat. Sol. B 247, 2924 (2010).

[10] See Supplemental Material.

[11] S. D. Ganichev, S. A. Emel'yanov, and I. D. Yaroshetskii, JETP Lett. 35, 368 (1982).

[12] S. D. Ganichev et al., Phys. Rev. Lett. 80, 2409 (1998).

[13] S. D. Ganichev and W. Prettl, Intense Terahertz Excitation of Semiconductors (Oxford Univ. Press, 2006).
[14] E. Ziemann et al., J. Appl. Phys. 87, 3843 (2000).

[15] B. E. A. Saleh and M. C. Teich, Fundamentals of Photonics (John Wiley \& Sons, Inc., 2007).

[16] J. Karch et al., Phys. Rev. Lett. 97, 182107 (2010).

[17] L. I. Magarill and M. V. Entin, Phys. Solid State 21, 743 (1979).

[18] V. L. Alperovich et al., JETP Lett. 31, 547 (1980).

[19] F. Speck et al., arXiv:1103.399v1.

[20] E. J. H Lee et al., Nature Nano. 3, 486 (2008).

[21] C. Virojanadara et al., Phys. Rev. B 78, 245403 (2008).

[22] A. Bostwick et al., Nature Phys. 3, 36 (2007).

[23] T. Ohta et al., Phys. Rev. Lett. 98206802 (2007).

[24] K. V. Emtsev et al., Phys. Rev. B. 77, 155303 (2008).

[25] S. Kopylov et al., Appl. Phys. Lett. 97, 112109 (2010).

[26] J. Eroms and D. Weiss, New J. Phys. 11, 095021 (2009).

[27] U. Stöberl et al., Appl. Phys. Lett. 93, 051906 (2008).

[28] W. Weber et al., Phys. Rev. B 77, 245304 (2008). 